\title{
AN ALGEBRAIC PROBLEM IN CONTROL THEORY
}

\author{
by ARTHUR WOUK $\dagger$ \\ (Received 29th April 1963)
}

1. In the series of papers in the early forties summarised in (1), (2), A. I. Lur'e showed how to utilise Liapunov's second or direct method in the investigation of the stability of linear automatic control systems with a single nonlinear actuator. His approach consists of

1. the transformation of the original system of differential equations via the so-called Lur'e transformation into canonical coordinates in which the construction of the Liapunov function is direct, and

2 . the conversion of the differential problem into a purely algebraic problem.

We will be concerned here with the questions of the existence and construction of the Lur's transformation.

A system with a single nonlinear actuator is described by a system of differential equations (cf. e.g. Hahn (5), p. 42):

$$
\left\{\begin{array}{l}
y^{\prime}=A y+u p, \\
u^{\prime}=f(s), s=(b, y)-h u, h>0,
\end{array}\right.
$$

where ' $=d / d t, A$ is a real constant $n \times n$ matrix, $b$ and $p \neq 0$ are real constant $n$-vectors, (,) denotes scalar product, and $f$ is a real continuous nonlinear function such that $s f(s) \geqq 0$. Here the vector $y$ represents the physical state of the system, the scalar $u$ represents the amount of control to be utilised and the vector $p$ represents the distribution of the control $u$ among the state variables. An error signal $s$ is constructed from the deviation of $y$ from a plane normal to the vector $b$; modified by a multiple of the current control $u$, it signals an actuator whose response is characterised by the non-linear function $f(s)$. In many physical systems, the ability to control state variables is limited to those state variables which represent physical velocities; only their derivatives may be changed (Newton's Laws of Motion). Thus a physically significant problem is one in which $A, p, f$ are assumed as given; the physically interesting problem of stability is that of selecting a vector $b$ such that the solution $y=0$ of (1) is asymptotically stable in the large (cf. e.g. (4), pp. 7-8) for all functions $f$ belonging to a suitable class of functions. (This means that all solutions of

$\dagger$ Applied Research Laboratory, Sylvania Electronic Systems, A division of Sylvania Electric Products, Inc., Waltham 54, Massachusetts. Now on leave at the Mathematics Research Center, U.S. Army, University of Wisconsin, Madison, Wisconsin.

Sponsored by the Mathematics Research Center, United States Army, Madison, Wisconsin under Contract No.: DA-11-022-ORD-2059.

E.M.S.-O 
(1) tend to zero as $t \rightarrow \infty$ ). It should be noted, however, that in many electronic systems, all the variables in the mathematical representation may be controlled.

We note first (compare Hahn (5), p. 43) that the transformation to the desired canonical coordinates is accomplished by a Lur'e transformation

$$
y=B x+u q,
$$

which converts (1) into the form

$$
\left\{\begin{array}{l}
x^{\prime}=D x+m f(s) \\
s^{\prime}=(c, x)-h f(s)
\end{array}\right.
$$

Here $D$ is the Jordan normal form of $A, c=B^{T} A^{T} b$ ( ${ }^{T}$ denotes transpose) and $m$ is a vector $\dagger$ with $m_{i}=1$ or $0, i=1, \ldots, n$. It is easy to see that we require $B$ to be a nonsingular matrix such that $B^{-1} A B=D$ and that $A q=-q$, $B^{-1} q=-m$, or

$$
A B m=p .
$$

The choice of $m_{i}$ as either 1 or 0 is a normalisation for the convenience of analysis and exposition. What is important is the vanishing or non-vanishing of $m_{i}$; should $m_{i}$ vanish, then the corresponding canonical variable $x_{i}$ is not directly controlled by the actuator. (It may, however, be indirectly controlled.) Lur'e (1) (see also (2), pp. 38-47) gives certain algorithms, valid for the case of a matrix with $n$ distinct characteristic values, in determinantal form, for what is, in effect, a construction of $B$ and $q$. In these determinantal algorithms, the geometric meaning of the manipulations is not clear, and the construction fails for the case of multiple characteristic values. We will exhibit a procedure which determines the possibility of construction of the Lur'e transformation as it constructs it; it will be seen that the algorithm of Lur'e valid for matrices with $n$ distinct characteristic values results in no more than the special scaling of an arbitrary $B$ which transforms $A$ to Jordan normal form so as to set $m_{i}=1$, or 0 . We will show that the question of which canonical variables can be controlled $\left(m_{i} \neq 0\right)$ can be resolved by knowing any matrix $B$ which reduces $A$ to Jordan normal form.

Recently, Yakubovich has shown that the transformation to canonical coordinates is unnecessary from the point of view of theory (cf. (3) pp. 75-103, in particular pp. 75-91 or (8), pp. 129-123 for expositions in English of his work.) That is to say, the conditions of Lur'e for the stability of the controlled system are derived by matrix theoretic methods which do not directly utilise the Jordan normal form. Hidden in the theory is the necessity for solving the matrix equation $A^{T} L+L A=-C$ for any prescribed positive definite symmetric matrix $C$. In fact this is a very difficult matter if $A$ is not in Jordan normal form. Thus, at the point of application it remains necessary (cf. e.g. LaSalle and Lefschetz (3) pp. 91-103 for the case where this form is diagonal), to utilise the transformation to canonical coordinates in order to establish

$\dagger$ Hahn ((5), p. 43, p. 138) first observed that it is not always possible to have $m_{i}=1$, $i=1, \ldots, n$; i.e. some zero values may be necessary if $p$ is orthogonal to characteristic vectors of $A$. 
computable constraints on the control variables $p, b$ and $h$ of (1) which lead to desired stability properties (in this regard, see also (4)). Thus it remains necessary to determine which sets of canonical variables may be achieved by this reduction, what effects, if any, this indeterminacy in the reduction has on stability, and which canonical variables permit easiest calculation of stability criteria.

Lastly, in an appendix we will relate these results to those of Kriuchkov (9) which construct a "canonical form", other than the Lur'e form, which need not preserve the stability properties of the original system. We will see that the Lur'e form is, in suitable modification, always achievable, and that stability is always preserved.

2. We note first that if we set $p=B \rho$, then (3) becomes $D m=\rho$. Thus, for a given $B$, the decomposition of $p$ along the basis consisting of the column vectors of $B, b_{1}, \ldots, b_{n}$, determines the possibility of finding $m$. If $m$ exists, it is unique up to a solution of the homogeneous equation $D m=0$, i.e. to an arbitrary vector in the null space of $D$ while $\rho$ must be orthogonal to the null space of $D$. Thus p must be orthogonal to the null space of $A$. Henceforth this is always assumed.

Next we note that if $\lambda_{i} \neq 0$ is a characteristic value of $A$ (and $D$ ) possessing two or more linearly independent characteristic vectors, then there exist two columns of $B, b_{i_{1}}, b_{i_{2}}$ satisfying $A b_{i_{j}}=\lambda_{i} b_{i_{j}}, j=1,2$. If $\rho_{i_{1}}$ and $\rho_{i_{2}}$ are both nonzero, then we must have $\rho_{i_{1}}=\rho_{i_{2}}$. To see this, note that $D m=\rho$ implies $\lambda_{i} m_{i j}=\rho_{i j}$. Since $\lambda_{i}, \rho_{i_{1}}$ and $\rho_{i_{2}}$ are not zero, then we must have $m_{i_{j}}=1$, $=1,2$ and $\rho_{i_{1}}=\rho_{i_{2}}=\lambda_{i}$. This, however, is only an apparent restriction, for we can always rescale $\rho$ by renormalising the characteristic vectors $b_{i_{1}}$, $b_{i_{2}}, \ldots$, so that $\rho_{i_{j}}=\left(b_{i}, p\right)$ is a constant for all characteristic vectors belonging to a given nonzero characteristic value. Note that this entails, with the renormalisation of $b_{i j}$, the renormalisation of the other columns of $B$ which are in the same cyclic invariant subspace as $b_{i j}$.

On a given cyclic invariant subspace corresponding to an elementary divisor $\left(\lambda-\lambda_{i}\right)^{k_{i}}, D$ has the representation

$$
\left(\begin{array}{ccccc}
\lambda_{i} & 1 & & & \\
& \lambda_{i} & 1 & & \\
& & \cdots & & \\
& & & \lambda_{i} & 1 \\
& & & & \lambda_{i}
\end{array}\right)
$$

If the relevant components of $m$ and $\rho$ are indexed by $j_{1}, \ldots, j_{k}$, we find that on this subspace (3) is equivalent to

$$
\begin{aligned}
& \lambda_{i} m_{j_{1}}+m_{j_{2}}=\rho_{j_{1}} \\
& \lambda_{i} m_{j_{2}}+m_{j_{2}}=\rho_{j_{2}} \\
& \ldots \\
& \lambda_{i} m_{j_{k-1}}+m_{j_{k}}=\rho_{j_{k-1}} \\
& \lambda_{i} m_{j_{k}}=\rho_{j_{k}} .
\end{aligned}
$$


From these equations, it becomes clear that (3) is not in general solvable for $m_{i}=1$ or 0 , and the construction of the transformation is not immediate. Further it seems that renormalisation of the $b_{j_{k}}$ might not effect equality of other $\rho_{i}$ 's belonging to distinct invariant subspaces corresponding to the same $\lambda_{i}$. It might appear from this that the restriction of $m_{i}$ to the values 1 or 0 is at fault here. If $m_{i}$ were allowed arbitrary values, then the numerical value of the $\rho_{i}$ would enter into the determination of the $m_{i}$; we shall see below that this is misleading: only the vanishing and non-vanishing of some of the $\rho_{i}$ are significant to this determination.

3. The starting point of our procedure is the observation that the transformation to Jordan form of $A$ is unique only to an arbitrary nonsingular matrix $V$ which commutes with $A$, for then $(V B)^{-1} A(V B)=B^{-1} A B=D$. Henceforth we assume that we know a matrix $B$ which reduces $A$ to $D$. The construction of such matrices $B$ is well known (cf. e.g. (6), pp. 66-69 or (7), pp. $159 \mathrm{ff}$.). We can restate our problem as the determination of a matrix $V$ which commutes with $A$ such that under the transformation $y=V B z+u q$ the system (1) goes over into (2) where $c=B^{T} V^{T} A^{T} b$, and in addition

$$
A V B m=p \text {. }
$$

Hence $q=-V B m$. (We omit components in the null space of $A$.)

In terms of the given nonsingular matrix $B, p$ can be decomposed uniquely as

$$
p=\sum_{i=1}^{n} \rho_{i} b_{i}=B \rho
$$

for a unique vector $\rho$, with components $\rho_{1}, \ldots, \rho_{n}$. Among the matrices which commute with $A$ are the polynomials in $A$, say

$$
g(A)=\sum_{i=1}^{n-1} \alpha_{i} A^{i}
$$

Unfortunately, if any two elementary divisors of $A$ have a nonlinear common factor, a suitable $V$ may be found in this way only for restricted classes of vectors $p$ (see below). Instead, we observe (cf. e.g. (6), pp. $143 \mathrm{ff}$. or (7), pp. 220-223) that the set of matrices $V$ which commute with $A$ is generated by $V=B U B^{-1}$ where $U$ runs over the set of matrices which commute with $D$. Then (5) can be rewritten as $B^{-1} A V B m=\rho$ or

$$
D U m=U D m=\rho .
$$

4. In order to construct the desired nonsingular matrix $V$, we must study the block structure of $U$, which is dependent upon the elementary divisors of $D$. If these are

$$
\left(\lambda-\lambda_{1}\right)^{r_{1}},\left(\lambda-\lambda_{2}\right)^{r_{2}}, \ldots,\left(\lambda-\lambda_{q}\right)^{r_{q}},
$$

then ((6), p. 144 or (7), p. 218) $U$ is decomposed into blocks $U_{\alpha \beta}, \alpha \beta=1, \ldots, q$ where $U_{\alpha \beta}$ is a rectangular matrix with $r_{\alpha}$ rows and $r_{\beta}$ columns. Further, 
$U_{\alpha \beta}$ is a zero matrix if $\lambda_{\alpha} \neq \lambda_{\beta}$, while it is " upper triangular" if $\lambda_{\alpha}=\lambda_{\beta}$ ("Upper triangular" for an $m$ row, $n$ column matrix $M$ means $M_{i j}=0$ if $j<i+\max (0, n-m)$; for square matrices this is the usual definition.). Lastly, the elements along each diagonal of $U_{\alpha \beta}$ are constant. Thus each block contains $\min \left(r_{\alpha}, r_{\beta}\right)$ arbitrary parameters. It would therefore appear that we have sufficient freedom to restrict the class of matrices $U$ among which we look for our solution, possibly to upper triangular matrices $\left(U_{\alpha \beta}=\right.$ the zero matrix if $\alpha>\beta$ ). It will be seen below, however, that this can be done only in special circumstances; this will explain in part our inability to find $U$ among the matrix polynomials in $D$.

We will prove first two lemmas, one guaranteeing the solvability of (6) for arbitrary $p$, the other guaranteeing the nonsingularity of $U$.

It is evident from the description that we can restrict our attention to the invariant subspaces associated with a given nonzero characteristic value, say $\lambda_{1}$, since $U$ and $D U$ are reduced to block diagonal form with respect to these subspaces. Let $W$ denote the block in $U$ which corresponds to $\lambda_{1}$. We rewrite the form of (6) on such a subspace as follows. Let $\left(\lambda-\lambda_{1}\right)^{r_{1}}, \ldots,\left(\lambda-\lambda_{1}\right)^{r_{k}}$, $r_{1} \geqq r_{2} \geqq \ldots r_{k} \geqq 1$ be the elementary divisors associated with $\lambda_{1}$. Let the index pair $[i, t]$ denote the index.

$$
\sum_{\beta=1}^{i-1} r_{\beta}+t
$$

where $t=1, \ldots, r_{i}, i=1,2, \ldots, k$. (The sum is taken as zero when $i=1$.) Let $\left(U_{\alpha \beta}\right)_{i, j}=z_{\alpha, \beta, i-j}$, where $i=1, \ldots r_{\alpha} ; j=1, \ldots r_{\beta}$ and $\alpha, \beta=1, \ldots, k$. Since $U_{\alpha \beta}$ is upper triangular, it follows that $z_{\alpha, \beta, i-j}$ must vanish if

$$
j<i+\max \left(0, r_{\beta}-r_{\alpha}\right) .
$$

On the other hand, the matrix product $D U=U D$ has the same block structure as $U$, with elements $z_{\alpha, \beta, i-j}^{\prime}=\lambda_{1} z_{\alpha, \beta, i-j}+z_{\alpha, \beta, i-j+1}$. (We extend the definition of $z_{\alpha, \beta, i-j}$ by setting it equal to zero if $i$ or $j$ are outside their respective ranges $1, \ldots, r_{\alpha}$ and $1, \ldots r_{\beta}$ ). Let $W^{\prime}$ be the block in $D U$ which corresponds to $W$. Then (6) becomes

$$
\sum_{\beta=1}^{k} \sum_{j=1}^{r_{\beta}} z_{a, \beta, i-j}^{\prime} m_{[\beta, j]}=\rho_{[\alpha, i]}, \quad i=1, \ldots, r_{a}, \quad \alpha=1, \ldots, k .
$$

If we take $m_{[\beta, j]}$ as given, and hopefully $\equiv 1$, then (7) is to be regarded as $r_{1}+\ldots+r_{k}$ equations in the unknowns $z_{\alpha, \beta, i-j}^{\prime}$. There are

$$
m=\sum_{\alpha=1}^{k} \sum_{\beta=1}^{k} \min \left(r_{\alpha}, r_{\beta}\right)=r_{1}+3 r_{2}+\ldots+(2 k-1) r_{k}
$$

such unknowns. Thus we have more unknowns than equations. Solutions exist if the equations are consistent. The consistency conditions may be expressed in terms of an auxiliary matrix $M$ which we determine thus: enumerate 
the $z^{\prime}$ variables so that

$$
\begin{aligned}
& z_{1}^{\prime}=z_{1,1,0}^{\prime}, \ldots, z_{r_{1}}^{\prime}=z_{1,1,1-r_{1}}^{\prime}, \\
& z_{r_{1}+1}^{\prime}=z_{1,2,0}^{\prime}, \ldots, z_{r_{1}+r_{2}}^{\prime}=z_{1,2,1-r_{2}}^{\prime}, \\
& \ldots \\
& z_{r_{1}+\ldots+r_{k-1}+1}^{\prime}=z_{1, k, 0}^{\prime}, \ldots, z_{r_{1}+\ldots+r_{k}}^{\prime}=z_{1, k, 1-r_{k}}^{\prime}, \\
& z_{r_{1}+\ldots+r_{k}+1}^{\prime}=z_{2,1,1-\left(r_{1}-r_{2}\right)}^{\prime}, \ldots, z_{r_{1}+\ldots+r_{k}+r_{2}}^{\prime}=z_{2,1,1-r_{1}}^{\prime}, \\
& z_{r_{1}+\ldots+r_{k}+r_{2}+1}^{\prime}=z_{2,2,0}^{\prime}, \ldots
\end{aligned}
$$

That is, we start with the upper left corner of the $W^{\prime}$ matrix and enumerate from left to right in each row, those elements $z_{\alpha, \beta, i-j}^{\prime}$ in the 1st, $\left(r_{1}+1\right)$ th, $\ldots$, $\left(r_{1}+\ldots+r_{k-1}+1\right)$ th rows for which $j^{\prime} \geqq 1+\max \left(0, r_{\beta}-r_{\alpha}\right)$. Then $M z^{\prime}=\rho$ where $M$ is a rectangular matrix of the following block form:

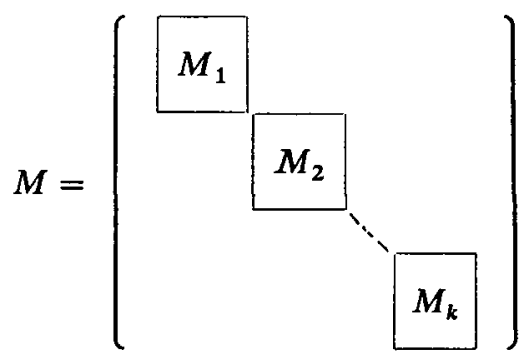

where $M_{i}$ is a matrix of $r_{i}$ rows, $i r_{1}+r_{i+1}+\ldots+r_{k}$ columns. If we subdivide $M_{i}$ into blocks of $r_{i}, \ldots, r_{i}, r_{i+1}, \ldots, r_{k}$ columns, then the $j$ th block has the form of the following $r_{i} \times r_{i}$ matrix

$$
\left[\begin{array}{llll}
m_{\left[j, r_{j}-r_{i}+1\right]} & & \ldots & m_{\left[j, r_{j}\right]} \\
m_{\left[j, r_{j}-1\right]} & m_{\left[j, r_{j}\right]} & 0 & \ldots \\
m_{\left[j, r_{j}\right]} & 0 & & \ldots
\end{array}\right]
$$

if $j \leqq i$, while it has the form

if $j \geqq i$.

$$
r_{i}-r_{j}\left(\begin{array}{lllll}
m_{[j, 1]} & m_{[j, 2]} & \ldots & & m_{\left[j, r_{j}\right]} \\
m_{[j, 2]} & & \ldots & m_{\left[j, r_{j}\right]} & 0 \\
m_{\left[j, r_{j}-1\right]} & m_{\left[j, r_{j}\right]} & 0 & \ldots & \\
m_{\left[j, r_{j}\right]} & 0 & \ldots & & \\
0 & \ldots & & & 0 \\
\vdots & & & & 0
\end{array}\right)
$$

From this structure, it follows that the assignment $m_{[\beta, t]} \equiv 1$ provides 
a matrix $M$ which is of maximal rank, since its rows constitute an independent set of $r_{1}+\ldots+r_{k}$ vectors in an $m$-dimensional space. In fact, it is sufficient for this if $m_{\left[1, r_{1}\right]} \neq 0$; then the 1st block of $M_{j}$ is a square nonsingular matrix. On the other hand, $m_{\left[1, r_{1}\right]}=0$ makes the rank of $M$ less than $r_{1}+\ldots+r_{k}$ so that the existence of $D U$ solving (6) is not guaranteed for arbitrary vectors $p$. We have proved the following:

Lemma 1. The condition $m_{\left[1, r_{1}\right]}=1$ is necessary and sufficient for the solvability of (7) for arbitrary $p$.

There are $\sum_{j=1}^{k}(2 j-2) r_{j}$ independent parameters in the $W^{\prime}$ matrix whose assignment leads to a solution of (7) with $m_{[\beta, j]} \equiv 1, \beta=1, \ldots, k, j=1, \ldots, r_{\beta}$. If, as would be desirable for computational purposes, the matrix $U$ is sought among the class of upper triangular matrices, the following change must be made in the preceding analysis. $W$ and $W^{\prime}$ are now upper triangular matrices and (7) specialises to

$$
\sum_{\beta=\alpha}^{k} \sum_{j=1}^{r \beta} z_{\alpha, \beta, i-j}^{\prime} m_{[\beta, j]}=\rho_{[\alpha, i]}, \quad i=1, \ldots, r_{\alpha}, \quad \alpha=1, \ldots, k .
$$

The number of unknowns $z_{\alpha, \beta, i-j}^{\prime}$ now is

$$
m=\sum_{\alpha=1}^{\beta} \sum_{\beta=1}^{k} \min \left(r_{\alpha}, r_{\beta}\right)=r_{1}+2 r_{2}+\ldots+k r_{k}
$$

which still exceeds the number of equations. We proceed as before in the enumeration of the elements of $z^{\prime}$, i.e. from left to right in $W^{\prime}$, but now

$$
\begin{aligned}
& z_{1}^{\prime}=z_{1,1,0}^{\prime}, \ldots, \\
& z_{r_{1}+r_{2}+\ldots+r_{k}+1}^{\prime}=z_{2,2,0}^{\prime}, \ldots, \\
& z_{r_{1}+2 r_{2}+\ldots+r_{k}+1}^{\prime}=z_{3,3}^{\prime}, \ldots,
\end{aligned}
$$

$M_{i}$ is now a rectangular matrix of $r_{i}$ rows, $r_{i}+r_{i+1}+\ldots+r_{k}$ columns whose $j$ th column block is given only by the second of the forms above, that for $j=i+p-1 \geqq i$. The assignment $m_{[\beta, \tau]} \equiv 1$ provides a matrix $M$ of maximal rank, as before. However, in this case $m_{[j, r j]}=1$ makes the first column block of $M_{j}$ a non-singular square matrix, while if any $m_{\left[j, r_{j}\right]}=0$, the corresponding $M_{j}$ has a row of zeros. We have then

Lemma 1'. $m_{\left[j, r_{j}\right]}=1, j=1, \ldots, k$ is necessary and sufficient for the solvability of $\left(7^{\prime}\right)$ for arbitrary $p$.

There are $\sum_{j=1}^{k}(j-1) r_{j}$ independent parameters in the $W^{\prime}$ matrix whose assignment leads to a solution of $\left(7^{\prime}\right)$ with $m_{[\beta, j]}=1, j=1, \ldots, r_{\beta}, \beta=1, \ldots, k$.

5. We wish next to guarantee the nonsingularity of $U$. Again, owing to the block structure of $U$ and $D U$, we work on the invariant subspace associated 
with $\lambda_{1} \neq 0$; there the nonsingularity of $D U$ and $U$ are equivalent. The singularity of $D U$ or $W^{\prime}$ there is equivalent to the existence of a set of numbers $\delta_{[\beta, j]}, \beta=1, \ldots, k, j=1, \ldots, r_{\beta}$, not all zero, such that

$$
\sum_{\beta=1}^{k} \sum_{j=1}^{r \beta} z_{\alpha, \beta, i-j}^{\prime} \delta_{[\beta, j]}=0, \quad i=1, \ldots, r_{\alpha}, \alpha=1, \ldots, k .
$$

Set $i=r_{\alpha}, \alpha=1, \ldots, k$, and consider the resulting $k$ equations

$$
\sum_{\beta=1}^{k} z_{\alpha, \beta, r_{\alpha}-r_{\beta}}^{\prime} \delta_{\left[\beta, r_{\beta}\right]}=0, \quad \alpha=1, \ldots, k .
$$

If the determinant of the auxiliary $k \times k$ matrix $\left(z_{\alpha, \beta, r_{\alpha}-r_{\beta}}^{\prime}\right)$ is not zero, then we must have $\delta_{\left[\beta, r_{\beta}\right]}=0, \beta=1, \ldots, k$, and (8) can be replaced by

$$
\sum_{\beta=1}^{k} \sum_{j=1}^{r_{\beta}-1} z_{\alpha, \beta, i-j}^{\prime} \delta_{[\beta, j]}=0, \quad i=1, \ldots, r_{\alpha}-1, \quad \alpha=1, \ldots, k .
$$

Now set $i=r_{\alpha}-1, \alpha=1, \ldots, k$. We obtain the system of equations

$$
\sum_{\beta=1}^{k} z_{\alpha, \beta, r_{\alpha}-r_{\beta}}^{\prime} \delta_{\left[\beta, r_{\beta}-1\right]}=0, \quad \alpha=1, \ldots, k-1 .
$$

The nonvanishing of the determinant of the auxiliary matrix again implies $\delta_{\left[\beta, r_{\beta}-1\right]}=0, \beta=1, \ldots, k$. We may proceed in this manner for exactly $r_{k}$ steps. Suppose that $r_{i}>r_{k}$ if $i=1, \ldots, s$ while $r_{i}=r_{k}$ if $i=s+1, \ldots, k$. Then it follows that $z_{\alpha, \beta, r_{\alpha}-r_{\beta}}^{\prime}=0$ if $\alpha \leqq s$ and $\beta \geqq s+1$. Then

$$
\operatorname{det}_{1 \leqq \alpha, \beta \leqq k}\left(z_{\alpha, \beta, r_{\alpha}-r_{\beta}}^{\prime}\right)=\operatorname{det}_{1 \leqq \alpha, \beta \leqq s}\left(z_{\alpha, \beta, r_{\alpha}-r_{\beta}}^{\prime}\right) \times \operatorname{det}_{s+1 \leqq \alpha, \beta \leqq k}\left(z_{\alpha, \beta, r_{\alpha}-r_{\beta}}^{\prime}\right) .
$$

Then at the end of $r_{k}$ steps the successors to equations (10) become

$$
\sum_{\beta=1}^{s} \sum_{j=1}^{r_{\beta}-r_{k}} z_{\alpha, \beta, i-j}^{\prime} \delta_{[\beta, j]}=0, \quad i=1, \ldots, r_{\alpha}-r_{k}, \quad \alpha=1, \ldots, s .
$$

Now only the $(s \times s)$ th principle minor of the auxiliary matrix remains. This is non-singular if and only if the auxiliary matrix is non-singular. We may proceed in this manner to show that if $\operatorname{det}\left(z_{\alpha, \beta, r_{\alpha}-r_{\beta}}^{\prime}\right) \neq 0$, then $W^{\prime}$ is nonsingular. On the other hand, if $\operatorname{det}\left(z_{\alpha, \beta, r_{\alpha}-r_{\beta}}^{\prime}\right)=0$ then (9) has a nonzero solution; then we can, by elementary column operations on $W^{\prime}$, construct a matrix $k$ of whose rows vanish, so that the matrix is singular. Since elementary column operations are themselves nonsingular, this proves

Lemma 2. The condition

$$
\operatorname{det}\left(z_{\alpha, \beta, r_{\alpha}-r_{\beta}}^{\prime}\right) \neq 0
$$

is necessary and sufficient for the nonsingularity of $W^{\prime}$.

If all $r_{\alpha}$ are distinct, so that $\left(z_{\alpha, \beta, r_{\alpha}-r_{\beta}}^{\prime}\right)$ is a lower triangular matrix, or else if we seek an upper triangular matrix, $U$, condition (11) becomes

$$
\prod_{\alpha=1}^{k} z_{\alpha, \alpha, 0}^{\prime} \neq 0
$$


6. Next we establish the conditions for the existence and nonsingularity of the matrix $U$. We may still restrict attention to the block $W^{\prime}$ in $D U$ corresponding to a single nonzero characteristic value $\lambda_{1}$. In (7), set $i=r_{\alpha}, \alpha=1$, $\ldots, k$. This yields the set of equations

$$
\sum_{\beta=1}^{k} z_{\alpha, \beta, r_{\alpha}-r_{\beta}}^{\prime} m_{\left[\beta, r_{\beta}\right]}=\rho_{\left[\alpha, r_{\alpha}\right]}, \quad \alpha=1, \ldots, k
$$

If $\rho_{\left[\alpha, r_{\alpha}\right]}=0, \alpha=1, \ldots, k$, then it is impossible to have any $m_{\left[\alpha, r_{\alpha}\right]} \neq 0$ and simultaneously, a nonsingular $z^{\prime}$-matrix (or, by Lemma 2 , a nonsingular matrix $\left.W^{\prime}\right)$. Thus we must set $m_{\left[\alpha, r_{\alpha}\right]}=0, \alpha=1, \ldots, k$ and can choose $z_{\alpha, \beta, r_{\alpha}-r_{\beta}}^{\prime}$ at will so as to satisfy (11).

Next consider the situation where $\rho_{\left[x, r_{x}\right]} \not \equiv 0$. Suppose first that $r_{1}>r_{2}$. Then $z_{1, \alpha, r_{1}-r_{k}}^{\prime}=0, \alpha,=2, \ldots, k$; if $\rho_{\left[1, r_{1}\right]} \neq 0$, then (12) may be solved for a nonsingular lower triangular $z^{\prime}$-matrix and a vector $m_{\left[\beta, r_{\beta}\right]}=1, \beta=1$, $\ldots, k$. We need only set $z_{1,1,0}^{\prime}=\rho_{\left[1, r_{1}\right]}$ and

$$
z_{\alpha, 1, r_{\alpha}-r_{1}}^{\prime}+z_{\alpha, a, 0}^{\prime}=\rho_{\left[\alpha, r_{\alpha}\right]}, \quad \alpha=1, \ldots, k,
$$

while all other $z_{\alpha, \beta, r_{\alpha}-r_{\beta}}^{\prime}=0$. This can always be done so that both (11) and (11') are satisfied and the matrix $W^{\prime}$ is nonsingular.

If $r_{1}=r_{2}=\ldots=r_{s}, s \leqq k$, and at least one of $\rho_{\left[\alpha, r_{\alpha}\right]} \neq 0,1 \leqq \alpha \leqq r$, then we may reorder the basis $b_{i}$ so that $\rho_{\left[1, r_{1}\right]}$ is again not zero, and proceed as above. It is also possible to have $m_{\left[1, r_{1}\right]}=1, m_{\left[\beta, r_{\beta}\right]}=0$ or 1 at will, $\beta=2$, $\ldots, k$, and preserve the nonsingularity of $W^{\prime}$. Thus to have $m_{\left[\beta, r_{\beta}\right]}=0$, set $z_{\beta, 1, r_{\beta}-r_{1}}^{\prime}=\rho_{\left[\beta, r_{\beta}\right]}, z_{\beta, \beta, 0}^{\prime} \neq 0$.

If, however, $\rho_{\left[\alpha, r_{\alpha}\right]}=0, \alpha=1, \ldots, s$, then we must set $m_{\left[\alpha, r_{\alpha}\right]}=0$,

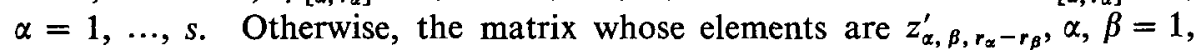
$\ldots, s$, would be singular. Since the other elements of the first $s$ rows of the $z^{\prime}$ matrix are zero, this implies the singularity of the $z^{\prime}$ matrix, and by Lemma 2 , the singularity of the $W^{\prime}$ matrix. When $m_{\left[\alpha, r_{\alpha}\right]}=\rho_{\left[\alpha, r_{\alpha}\right]}=0, \alpha=1, \ldots, s$,

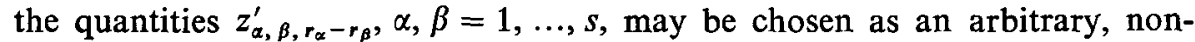
singular $s \times s$ matrix. The question of the nonsingularity of the $z^{\prime}$ matrix is now reduced to that of the nonsingularity of the $k-s \times k-s$ matrix $z_{\alpha, \beta}^{\prime}, r_{\alpha}-r_{\beta}$ with $\alpha, \beta=r+1, \ldots, k$. The argument above applies without exception to this submatrix.

Now suppose that $m_{\left[\beta, r_{\beta}\right]}, \beta=1, \ldots, k$, and the nonsingular auxiliary $k \times k$ matrix $\left(z_{\left.\alpha, \beta, r_{\alpha}-r_{\beta}\right)}^{\prime}\right.$ have been chosen. The remaining equations of (7) can be written as

$$
\sum_{\beta=1}^{k^{\prime}} \sum_{j=1}^{r_{\beta}-1} z_{a, \beta, i-j}^{\prime} m_{[\beta, j]}=\rho_{[\alpha, i]}-\rho_{\left[\alpha, r_{\alpha}\right]}, \quad i=1, \ldots, r_{\alpha}-1, \ldots, k^{\prime}, \ldots \text { (13) }
$$

where $k^{\prime} \leqq k$ is such that $r_{\alpha}=1, k^{\prime}<\alpha \leqq k$. With appropriate identification of terms, this system is of the same form as (7). Hence Lemma 1 may be applied to (13). We conclude that the assignment

$$
m_{\left[\beta, r_{\beta}-1\right]}=1, \quad \beta=1, \ldots, k^{\prime}
$$


is necessary and sufficient for the solvability of (13) for any vector. The remaining elements of the $m$-vector may be chosen as 0 or 1 at will.

We summarise these results as follows:

Let $\lambda_{1} \neq 0$ be a characteristic value of $A$ with elementary divisors $\left(\lambda-\lambda_{1}\right)^{r_{1}}$, $\ldots,\left(\lambda-\lambda_{1}\right)^{r_{k}}, r_{1} \geqq r_{2} \geqq \ldots \geqq r_{k} \geqq 1$ and characteristic vectors $b_{1}, \ldots, b_{k}$. Let $\left(b_{i}, p\right)=0, i=1, \ldots, s-1$ while $\left(b_{s}, p\right) \neq 0$. Let

$$
r_{\alpha_{s}-1}>r_{\alpha_{s}}=r_{\alpha_{s}+1}=\ldots=r_{s}=\ldots=r_{t_{s}}>r_{t_{s}+1} \text {. }
$$

Then the assignment

$$
\begin{aligned}
& m_{\left[\alpha, r_{\alpha}\right]}=0, \quad \alpha=1, \ldots, \alpha_{s}-1, \\
& m_{\left[\alpha_{s}, r_{\alpha_{s}}\right]}=1,
\end{aligned}
$$

assures the existence and permits the calculation of a nonsingular matrix $U$ which commutes with $D$ and satisfies (6). The remaining elements of $m$ may be taken as 1 or 0 at will.

The choice of $m_{\left[\alpha_{s}, r_{\left.\alpha_{s}\right]}\right.}$ in the above statement is not essential; any one of $m_{\left[\alpha, r_{\alpha}\right]}$ could be chosen to be $1, a_{s} \leqq \alpha \leqq t_{s}$ and the statement remains true. Further, at least one of them must be so chosen.

The matrix $U$ can be chosen among the upper triangular matrices if and only if $\left(b_{i}, p\right) \neq 0$ for at least one characteristic vector $b_{i}$ belonging to each set of elementary divisors of given exponent. To see this, consider again equations $\left(7^{\prime}\right)$. Here $z_{\alpha, \beta, r_{2}-r_{\beta}}^{\prime}=0$ if $r_{\alpha} \neq r_{\beta}$. Repeating the preceding analysis leads to this form of the assertion, using Lemma $1^{\prime}$ in the place of Lemma 1. Lastly, if the elementary divisors have no nonlinear common factors, then $U$ may be realised as a polynomial $g(D)$, after renormalisation of the corresponding characteristic vectors. To see this, note that this implies that $U$ may be taken as a block diagonal matrix, each block of which is precisely of the form which is realisable by a suitable interpolating polynomial in $D$ (see, for instance, (6), pp. 62-63 or (7), p. 100 . If the elementary divisors are all linear, then $D$ is diagonal and $U$ is determined by (6) as a diagonal matrix, used only for scaling purposes.

Thus the particular transformations described by Lur'e are seen to effect only the scaling of $m_{i}$, when they are correct, i.e. in the case that $A$ has $n$ distinct characteristic roots.

The preceding treatment leads to complex transformations, if the matrix $A$ has complex characteristic values. Since $A x=\lambda x+y$ if and only if

$$
A x^{*}=\lambda^{*} x^{*}+y^{*}
$$

(* denotes complex conjugate) when $A$ is real, the equivalent real transformations can be constructed which lead to the real Jordan normal form, in the usual manner. The reality of $p$ and $b$ implies that all operations associated with the characteristic value $\lambda$ are exactly paralleled in the treatment of $\lambda^{*}$, and the final form (2) can be chosen with the control $f(s)$ present $\left(m_{i}=1\right)$ in precisely one pair of equations belonging to each pair of characteristic 
values $\lambda, \lambda^{*}$, for which $\left(p, b_{i}\right) \neq 0$ for some non-zero characteristic vector $b_{i}$ belonging to $\lambda$.

7. The indeterminacy in the form of $m$ allows us to raise two questions. Firstly, does this indeterminacy lead to different stability criteria? Secondly, does this indeterminacy permit simplified calculation of conditions which guarantee absolute stability? The first question is answered as follows. Suppose that a matrix $B$ has been calculated and the equations are in a real Jordan normal form (2); let another canonical form

$$
\left\{\begin{array}{l}
\bar{x}^{\prime}=D \bar{x}+\bar{m} f(s) \\
s^{\prime}=(\bar{c}, \bar{x})-h f(s)
\end{array}\right.
$$

be achieved by the matrix $U$ where $U$ commutes with $D$ and is non-singular. Then $\bar{m}=U^{-1} m$ and $\bar{c}=U^{T} c$. The Lur'e special Liapunov function implies absolute stability if and only if

$$
h>\left(\Delta^{-1}\left[L m+\frac{1}{2} c\right], \quad\left[L m+\frac{1}{2} c\right]\right)
$$

where $\Delta$ is some real symmetric positive definite $n \times n$ matrix and $L$ is the unique real symmetric positive definite solution of the matrix equation $D^{T} L+L D=-\Delta$ (cf. (3), p. 85 or (8), p. 122). Then

$$
\left(\Delta^{-1}\left[L \bar{m}+\frac{1}{2} \bar{c}\right],\left[L \bar{m}+\frac{1}{2} \bar{c}\right]\right)=\left(\left(U^{T} \Delta U\right)^{-1}\left[\left(U^{T} L U\right) m+\frac{1}{2} c\right],\left[\left(U^{T} L U\right) m+\frac{1}{2} c\right]\right),
$$

while

$$
D^{T}\left(U^{T} L U\right)+\left(U^{T} L U\right) D=-U^{T} \Delta U
$$

From this we conclude that a triple $(h, m, c)$ makes (2) absolutely stable if and only if the triple $(h, \bar{m}, \bar{c})$ makes $\left(2^{\prime}\right)$ absolutely stable. This answers the first question negatively. The second question can be answered affirmatively. Notice first that the stability criterion (14) depends upon the calculation of the solution of

$$
D^{T} L+L D=-\Delta
$$

this is not readily calculable for arbitrary positive definite matrices. Even for $3 \times 3$ matrices the computations are too cumbersome unless, as is customary, we make $\Delta$ a diagonal matrix. This yields sufficient conditions for (14) which in turn is only sufficient for absolute stability. If we solve (14) for all sets of $h$ and $c$ which satisfy (14) for a given $m$ and some positive definite diagonal matrix $\Delta$, we obtain stability criteria which depend upon the form of $m$. If $\lambda$ is a characteristic number with many elementary divisors, and $m$ has many non-zero components, this leads to cumbersome calculations. If we replace $m$ by $\bar{m}$, with only one non-zero component for each distinct characteristic number $\lambda$, the work is easier. As an example, suppose that $D$ is

$$
\left[\begin{array}{lll}
\lambda & 1 & 0 \\
0 & \lambda & 0 \\
0 & 0 & \lambda
\end{array}\right]
$$


where $\lambda<0$. Then (15) becomes

$2 \lambda\left[\begin{array}{lll}l_{11} & l_{12} & l_{13} \\ l_{21} & l_{22} & l_{23} \\ l_{31} & l_{32} & l_{33}\end{array}\right]+\left[\begin{array}{lrl}0 & l_{11} & 0 \\ l_{11} & 2 l_{12} & l_{13} \\ 0 & l_{13} & 0\end{array}\right]=\left[\begin{array}{ccc}-\delta_{1} & 0 & 0 \\ 0 & -\delta_{2} & 0 \\ 0 & 0 & -\delta_{3}\end{array}\right]$.

Then $L$ is

$$
\left[\begin{array}{ccc}
-\delta_{1} / 2 \lambda & \delta_{1} / 4 \lambda^{2} & 0 \\
\delta_{1} / 4 \lambda^{2} & -\delta_{2} / 2 \lambda-\delta_{1} / 4 \lambda^{3} & 0 \\
0 & 0 & -\delta_{3} / 2 \lambda
\end{array}\right]
$$

If $\bar{m}_{1}=0, \bar{m}_{2}=1, \bar{m}_{3}=0$, then (14) becomes

$$
h>\delta_{1}^{-1}\left(\delta_{1} / 4 \lambda^{2}+\bar{c}_{1} / 2\right)^{2}+\delta_{2}^{-1}\left(-\delta_{2} / 2 \lambda-\delta_{1} / 4 \lambda^{3}+\bar{c}_{2} / 2\right)^{2}+\delta_{3}^{-1}\left(\bar{c}_{3} / 2\right)^{2} .
$$

Clearly $\bar{c}_{3}$ is unrestricted. If both $\bar{c}_{1}$ and $\bar{c}_{2}$ are non-positive, the right side can be made arbitrarily small for suitable choice of $\Delta$. If $\bar{c}_{2}>0$, then for any. $\delta_{1}$ the second term is minimal if $\delta_{2}=\delta_{1} / 2 \lambda^{2}-\lambda \bar{c}_{2}>0$ with minimal value $\delta_{1} / 2 \lambda^{2}-\bar{c}_{2} / \lambda$. Thus sufficient for absolute stability is

$$
h>9 \delta_{1} / 16 \lambda^{4}+\bar{c}_{1}^{2} / 4 \delta_{1}+\bar{c}_{1} / 4 \lambda^{2}-\bar{c}_{2} / \lambda .
$$

If $\delta_{1}=2 \lambda^{2}\left|\bar{c}_{1}\right| / 3$ the right side is again minimised and a stability criterion can be written as

$$
h>\varepsilon\left(c_{2}\right)\left\{\frac{3\left|\bar{c}_{1}\right|+\bar{c}_{1}}{4 \lambda^{2}}-\frac{\bar{c}_{2}}{\lambda}\right\}
$$

where $\varepsilon(t)=0$ if $t \leqq 0$, while $\varepsilon=1$ if $t>0$. Suppose now that the reduction of (1) to (2) had yielded $m_{1}=m_{2}=m_{3}=1$. Then $U$ can be taken as

since $\bar{c}=U^{T} c$, we have

$$
\left[\begin{array}{lll}
1 & 1 & 0 \\
0 & 1 & 0 \\
0 & 1 & 1
\end{array}\right]
$$

$$
\begin{aligned}
& \bar{c}_{1}=c_{1}, \\
& \bar{c}_{2}=c_{1}+c_{2}+c_{3}, \\
& \bar{c}_{3}=c_{3},
\end{aligned}
$$

and a sufficient condition for absolute stability is

$$
h>\varepsilon\left(c_{1}+c_{2}+c_{3}\right)\left\{\frac{3\left|c_{1}\right|+c_{1}}{4 \lambda^{2}}-\frac{c_{1}+c_{2}+c_{3}}{\lambda}\right\} .
$$

In the present case the saving in effort is small but noticeable. If the exponents of the various elementary divisors had been higher, the saving would have been more significant.

\section{Appendix}

Kriuchkov (9) (in our notation) represents the fundamental matrix $e^{A t}$ of the homogeneous system $y^{\prime}=A y$ as a linear combination of the terms of 
the form $t^{j} e^{\lambda_{k} t}$ with matrix coefficients which are the so-called components of $A$. In this form, the highest power $t^{j}$ which appears is one less than the power of $\left(\lambda-\lambda_{k}\right)$ which appears in the minimal polynomial of $A$ (cf. e.g. (5), pp. $110 \mathrm{ff}$.). If $k$ is the degree of the minimal polynomial, then the solution of the system

$$
\left\{\begin{array}{l}
y^{\prime}=A y+u p \\
u=f(s), s=(b, y)
\end{array}\right.
$$

is represented, using the variation of constants formula, as

$$
y=e^{A t} y_{0}+G \zeta
$$

where $G$ is a certain constant $n \times k$ matrix and $\xi$ is a particular solution of

$$
\xi^{\prime}=\Lambda \xi+m f(s) \text {. }
$$

Here $\Lambda$ is a $k \times k$ matrix in Jordan normal form whose characteristic (and minimal) polynomial is the minimum polynomial of $A$, while the projection of $m$ on each invariant subspace of $\Lambda$ has component one along the characteristic vector and zero elsewhere. Should $\lambda_{i}$ be a characteristic root with positive real part, of greater multiplicity in the characteristic equation than in the minimal equation of $A$ the stability of (17) does not imply the stability of (16). This, however, is not the Lur'e transformation, as constructed above. The calculations above show that there is a vector $m$, and nonsingular matrices $B$ and $V$ such that $y=V B z$ transforms (16) into

$$
\left\{\begin{aligned}
z^{\prime} & =D z+m f(s) \\
s & =(c, z)
\end{aligned}\right.
$$

where $D=B^{-1} A B, c=B^{T} V^{T} b$, and $V$ is a matrix which commutes with $A$. In this instance, equation (5) becomes

$$
V B m=p .
$$

Setting

we obtain

$$
V=B U B^{-1} \text {, }
$$

$$
U m=\rho,
$$

where $U$ is an arbitrary matrix commuting with $D$. It is easy to see that the construction used for $D U$ is now a construction for $U$. This Lur'e transformation, however, clearly preserves stability and instability properties.

I wish to thank Mr P. C. Parks for his valuable comments on an earlier version of this paper.

\section{REFERENCES}

(1) A. I. LUR'E, Some nonlinear problems in the theory of automatic control (Gos. Isdat. Tekh. Theor. Lit. Moscow, 1951; available in English from Her Majesty's Stationery Office, London (1957)). 
(2) A. M. Letov, Stability in nonlinear control systems (Princeton University Press, Princeton, New Jersey), pp. 33-47.

(3) J. LA SAlle and S. LefsCheTz, Stability by Liapunov's direct method (Academic Press, New York, 1961).

(4) J. E. Gibson, E. S. McVey, C. D. Leedham, Z. V. ReKasius, D. G. Schultz and R. SRIDHER, Stability of nonlinear control systems by the second method of Liapunov (AFMDC-TR-61-6, Vol. II, U.S. Dept. of Commerce, Office of Technical Services, Washington 25, D.C., 1961).

(5) W. HAHN, Theorie und Anwendung der Direkten Methode von Liapunov (Springer, Berlin, 1959).

(6) H. W. Turnbull and A. C. AItKen, The theory of canonical matrices (Blackie and Sons, London, second edition, 1948).

(7) F. R. Gantmacher, The theory of matrices, Vol. I (Chelsea Publishing Co., New York, 1959).

(8) N. N. KrasovskiI, Stability of motion (Stanford University Press, Stanford, California, 1963).

(9) N. B. KRIUCHKOV, Investigation of a peculiarity of nonlinear equations of control systems in the case of multiple roots by application of the theory of matrices, PMM Vol. 24, No. 4 (1960), 749-754, translated in Applied Mathematics and Mech. Vol. 24, No. 4 (1960), 1125-1134.

\section{Mathematics Research Center \\ MADISON, WISCONSIN}

